\title{
SISTEMA PRIVADO DE PENSIONES
}

PRIVATE PENSION SYSTEM

Zelma Wong Torres*

Docente Principal de la Facultad de Ciencias Contables

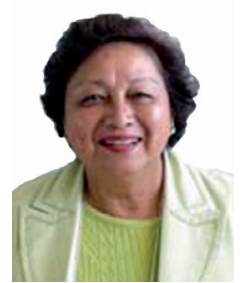

Universidad Nacional Mayor de San Marcos-UNMSM / Lima-Perú

[Recepción: Julio de 2013/ Conformidad: Agosto 2013]

\section{RESUMEN}

El presente artículo, se basa en el Estudio de Investigación denominado "Implementación de un adecuado Control Interno al Sistema Privado de Pensiones (AFP)", donde se determina que se debe estudiar a profundidad este sistema, el cual ha sido tomado del modelo chileno y que se le conoce que va camino al fracaso. Por lo tanto, es necesario alertar a los afiliados para que tomen una mejor decisión, afiliándose al sistema que considere se ajuste a sus perspectivas $y$ aspiraciones. Es posible, describir la situación actual del régimen pensionario y resaltar cada una de las características; para lograr que los trabajadores decidan racionalmente y opten por elegir el que considere conveniente, así mismo, que las medidas tomadas por el Poder Ejecutivo Peruano sirvan para subsanar la problemática del Sistema Privado de Pensiones; entre ellos, proteger a los futuros jubilados, porque contribuyendo a su desarrollo, se logrará reducir la pobreza y brindar la atención en su vejez.

\section{Palabras Clave:}

Afiliados, monopolio, Sistema Privado de Pensiones, comisiones, seguros.

\section{ABSTRACT}

This article is based on research study entitled "Implementation of an appropriate internal control for private pensions systems (AFP) “, where it is determined to be studied in depth this system, which has been taken from the Chilean model and it is known that is poised to fail. Therefore, it is necessary to alert members to take a better decision, consider joining the system that suits our prospects and aspirations. It is possible, describe the current situation of the pension system and highlight each of the features, to ensure that workers decide rationally and choose to choose it deems appropriate, also, that the measures taken by the Peruvian Executive Power to serve as a remedy for the problems of the private pension system, among them protect future retirees, because its contributing for its development, poverty reduction will be achieved and provide care in their old age.

\section{Keywords:}

Affiliate, monopoly, Private Pension System, commissions, insurance.

\footnotetext{
* Doctora en Contabilidad, USP. Contadora Pública Colegiada Certificada. UNMSM. Email: zwongt@hotmail.com
} 


\section{INTRODUCCIÓN}

El Sistema Privado de Pensiones (SPP) fue creado mediante el Decreto Ley $N^{\circ} 25897$ de fecha 06 de diciembre de 1992, desde su inicio a la fecha, ha crecido en número de afiliados. Sin embargo, este crecimiento ha estado por debajo de lo inicialmente esperado.

Actualmente, el SPP peruano afronta problemas y limitaciones para su desarrollo, los que se reflejan en la baja afiliación al sistema de la fuerza laboral; así como, los altos costos administrativos y el bajo retorno de los fondos administrados. Estos factores están mutuamente vinculados, ya que una baja cobertura determina mayores costos administrativos por pensionista; por lo tanto, una baja rentabilidad neta para el afiliado.

Para dar cuenta de esta evolución y de las posibilidades de desarrollo del sistema previsional, el presente trabajo delinea brevemente su estructura y alcance.

\section{PLANTEAMIENTO DEL PROBLEMA}

¿Es óptimo, el control interno del Sistema Privado de Pensiones en el Perú?

\section{OBJETIVO GENERAL}

- Elaborar un adecuado control interno, a fin de que las AFP maximicen su rentabilidad a favor del afiliado.

\section{OBJETIVO ESPECÍFICOS}

- Proporcionar conocimiento del sistema privado de pensionarios para que cada trabajador decida a qué sistema debe afiliarse.

- Analizar los principales aspectos de la problemática del Sistema Privado de Pensiones (SPP).

- Presentar una descripción de la actual situación de dicho régimen pensionario y resaltar cada una de sus características.

- Revisar las últimas medidas emprendidas por el Poder Ejecutivo Peruano.

\section{POBLACIÓN AFILIADA AL SPP}

El SPP afilia a los trabajadores del sector privado y del sector público, centrándose a captar trabajadores sujetos al régimen de la actividad privada. En el SPP, cada trabajador elige la AFP para que administre sus aportes.

\section{PROBLEMÁTICA EN EL SPP}

Dentro de la problemática de los sistemas de pensiones en el Perú, deben distinguirse los aspectos referidos al afiliado, sea este público o privado.

Siendo un régimen de capitalización individual basado en un esquema de ahorro, no posee aparentemente desequilibrios financieros, pues cada afiliado financia su propia pensión. No obstante, por ser relativamente joven, el proceso de transición a dicho sistema requiere de la emisión y pago de Bonos de Reconocimiento y Bonos Complementarios, medidas que están orientadas a su consolidación. Una de las principales dificultades que enfrenta el SPP es que los beneficios que ofrece han sido insuficientes, por tal motivo, muchos afiliados demandan su desafiliación.

\section{SISTEMA NACIONAL DE PENSIONES}

El Sistema Nacional de Pensiones fue creado el 01 de mayo de 1973 con el Decreto Ley N ${ }^{\circ} 19990$ por el gobierno militar de Juan Velazco Alvarado, quien agrupó a las antiguas Caja Nacional del Seguro Social Obrero (Ley No 8433 y Ley N 13640 ) y la Caja del Seguro Social del Empleado (Ley $\mathrm{N}^{\circ} 13724$ ), que agrupaban a su vez, dos tipos de trabajadores: Obreros y Empleados, respectivamente. Asimismo, absorbió al Fondo Especial de Jubilación de Empleados Particulares (Decreto Ley $\mathrm{N}^{\circ} 17262$ ) que agrupaba a aquellos empleados del sector público, sujetos al régimen laboral de la actividad privada.

Desde entonces, se incorporó por primera vez a trabajadores independientes y se igualó el trato pensionario de los trabajadores obreros y empleados, bajo las mismas condiciones de edad y años de aportación.

El Sistema Nacional de Pensiones es un sistema de reparto, el cual tiene como característica principal el otorgamiento de prestaciones fijas (sobre contri-

72/ QUipUiramavoc | Vol. 21(40) 2013 
buciones no definidas) en valor suficiente para que la aportación colectiva de los trabajadores financie las pensiones.

En la actualidad, este sistema es administrado por la Oficina de Normalización Previsional (ONP).

Las prestaciones que otorga el SNP son cinco:

1. Invalidez

2. Jubilación

3. Viudez

4. Orfandad

5. Ascendencia

\section{SISTEMA NACIONAL DE PENSIONES}

\section{HISTORIA Y DESCRIPCIÓN}

Se ha establecido que el Sistema Privado de Administración de Fondos de Pensiones tiene como objetivo contribuir al desarrollo y fortalecimiento del Sistema de Previsión Social en el área de pensiones y está conformado por las Administradoras Privadas de Fondos de Pensiones (AFP) que otorgan obligatoriamente a sus afiliados las prestaciones de jubilación, invalidez, sobrevivencia y gastos de sepelio.

El Sistema Privado de Pensiones funciona bajo la modalidad de Cuentas Individuales de Capitalización.

\section{El Sistema Privado de Pensiones (SPP) en EL MUNDO}

Este sistema ha penetrado inclusive en países tradicionalmente de bienestar y en los que el Estado soporta una enorme carga previsional y de asistencia social, tales como Estados Unidos y Suecia, funcionando también en Hong-Kong.

En los 21 países que tienen un régimen privado de pensiones, existe un total de más de 88 millones de afiliados que acumulan en sus cuentas de ahorro individual fondos por más de 200 mil millones de dólares.

En América Latina, en donde existen alrededor de 55 millones de afiliados, su número creció y los fondos previsionales privados ascienden a casi 100 mil millones de dólares. En general, en todos los países en que existe el Sistema Privado de Pensiones, hay un marcado aumento del número de afiliados, por ello, es importante señalar que la reforma previsional tiene un alcance fundamental para otorgar más poder a la sociedad civil, a fin de desplazar al Estado de las decisiones que en la mayoría de los casos son erradas, respecto al manejo y destino de los fondos. El Sistema Nacional de Pensiones está en bancarrota como consecuencia de su concepción original, por desplazar hacia el Estado, los derechos de su manejo que mayormente ha sido de tipo político. Fondos que eran manejados para encubrir los déficits fiscales.

Es cierto, que la mayoría de los trabajadores no tienen cobertura formal, obligando al Estado a actuar de buen samaritano con todos los que quedan al margen, pero también, la ineficacia del Estado ha marcado un alto costo a esta realidad.

\section{Sistema Privado de Pensiones en el perú}

Como lo relatan Eduardo Marón y Eliana Carranza en «Diez Años del Sistema Privado de PensionesAvances, Retos $y$ Reformas» (Centro de Investigación Universidad del Pacífico, Lima, 2003), "la reforma que se instala en el Perú es el sistema Privado de Pensiones que surge como consecuencia del desequilibrio financiero del antiguo sistema -cuya tendencia de largo plazo era reforzada por el cambio en el patrón de envejecimiento de la población-, la presencia de incentivos hacia la distorsión de los beneficios percibidos por los jubilados -existía discriminación por género, grupos ocupacionales y generacionales-, y sobre todo se buscaba evitar que los ahorros previsionales de los trabajadores queden ampliamente expuestos a riesgos asociados a factores políticos".

Este tipo de funcionamiento como es conocido, no logró satisfacer a políticos que basaban su clientela electoral en el manejo de promesas y beneficios asociados a este tipo de derechos sociales; por ejemplo, la Ley N' 20530 fue perforada dieciocho veces por leyes clientelistas, sin embargo, se logró instalar y mantener el sistema hasta hoy.

Es frecuente escuchar la necesidad de migrar del sistema privado al público y viceversa, de la solidaridad y de la participación laboral en la marcha del sistema de pensiones está el triunfo del afiliado. 
No debe existir un trabajador que no tenga un sistema de seguridad social. Tampoco tiene sentido la migración entre el sistema privado y el público, no solamente por su distinta naturaleza (uno es de capitalización individual y el otro de reparto), sino porque no habría forma de liquidar la cuenta individual de un trabajador sin causarle un perjuicio no solo al modificar su naturaleza individual privada y de capitalización para convertirlo en un fondo solidario de propiedad del Estado.

\section{Capital mínimo de una AFP}

El capital mínimo de inicio de las AFP fueron de S/.5’000,000.00, que debe ser íntegramente suscrito $y$ totalmente pagado en dinero en el momento de su constitución.

La suma del capital se actualiza anualmente al cierre de cada ejercicio en función al índice de Precios al Consumidor para Lima Metropolitana que publica periódicamente el INEI o el indicador que lo sustituya. La base del referido índice, es el número que arroje para el mes de enero de 1992.

Sin perjuicio de lo establecido en el presente artículo, las AFP deben aumentar su capital social de acuerdo a los requerimientos de su operación con la exclusiva finalidad de proteger los derechos de los afiliados. Los respectivos aportes deben ser necesariamente en dinero. Antes de que las AFP inicien operaciones con el público deben tener inscritas y registradas en la bolsa las acciones representativas.

\section{Administración del Fondo de Pensiones}

Cada AFP, administra un Fondo de Pensiones (el Fondo), salvo lo dispuesto por el artículo $71^{\circ} \mathrm{del}$ Decreto Supremo No 054-97-EF. El Fondo no integra el patrimonio de las AFP y su contabilidad debe ser llevada por separado.

\section{SUPERINTENDENCIA DE BANCA Y SEguros (SBS)}

Objetivo: La SBS y AFP ejercen en representación del Estado, la función de control de las AFP.

Características:
Es una institución pública descentralizada del Sector Economía y Finanzas, con personería de derecho público interno y con autonomía administrativa, funcional y financiera. Aprueba y supervisa la ejecución de su propio presupuesto.

La SBS está presidida por un superintendente que es nombrado por el Presidente de la República, a propuesta del Consejo de Ministros, por un período de cinco años.

Se financia, entre otros, con:

- Créditos o donaciones de las instituciones o empresas no sujetas a la supervisión; recursos propios de las contribuciones por el derecho de supervisión que pagan las AFP.

- Otros ingresos. Las decisiones de SBS sobre asuntos de carácter general o particular, deben estar contenidas en resoluciones que serán publicadas en el "Diario Oficial" para su plena vigencia. Estas resoluciones constituyen precedentes administrativos de obligatoria observancia.

Principales atribuciones y obligaciones:

La SBS y AFP tienen las funciones siguientes:

Velar por la seguridad y la adecuada rentabilidad de los fondos que administran las AFP.

- Autorizar la organización y el funcionamiento de las AFP, y disolverlas o suspenderlas.

- Reglamentar el funcionamiento de las AFP y el otorgamiento de las prestaciones que brindan.

- Interpretar sujetándose a principios de derecho, las normas legales que rigen al SPP y a las AFP.

- Fiscalizar la inversión de los recursos de los fondos de pensiones complementarios de longevidad y del encaje legal.

- Establecer el plan de cuentas de la AFP y de los fondos.

- Desarrollar programas de capacitación, información y divulgación sobre SPP.

- Es responsable de difundir permanentemente, por medios masivos de comunicación social, los principales indicadores de resultados del SPP. 
Cuando existan a criterio del superintendente, evidencias de hechos que puedan incidir negativamente, en la situación económica o financiera de la AFP o del fondo, a solicitud de éste, el directorio convocará a Junta General de Accionistas. Asimismo, tienen derecho de asistir a las juntas, el Superintendente o los representantes que éste designe.

\section{Estado de Cuentas del Afiliado - AfP}

Las AFP remitirán cuatrimestralmente al domicilio del afiliado u otra dirección que éste comunique el ESTADO DE CUENTAS DEL AFILIADO-AFP.

\section{Prestaciones que otorga el Sistema Priva- do de Pensiones}

Los trabajadores que se encuentren incorporados al SPP, mediante su afiliación a una AFP, tienen derecho a las prestaciones que ofrece el SPP. Los beneficiarios de los afiliados activos o pasivos fallecidos también tienen derecho de corresponderles las prestaciones de sobrevivencia.

\section{Tipos de Prestaciones:}

De acuerdo a las normas legales vigentes, las prestaciones que se otorgan en el SPP son:

- Jubilación

- Invalidez

- Sobrevivencia

- Gasto de sepelio

El afiliado del SPP o sus beneficiarios sobrevivientes pueden optar por cualquiera de las siguientes modalidades de pensión, según corresponda:

- Retiro programado.

- Renta vitalicia familiar.

- Renta vitalicia Personal.

- Renta temporal con renta vitalicia diferida.

\section{Marco legal del Sistema Privado de Pensiones}

El Sistema Privado de Fondos de Pensiones (SPP) fue creado por Ley No 25897 el 6 de diciembre de 1992, para beneficio de los trabajadores dependientes, e independientes, siendo sus principalescaracterísticas:

- La capitalización individual de los aportes de sus afiliados.

- La libre afiliación.

- La administración del fondo por entidades especializadas y exclusivas denominadas Administradoras de Fondos de Pensiones (AFP).

- La regulación y fiscalización minuciosa del sistema por parte del Estado, la cual se efectúa a través de la Superintendencia de Banca y Seguros.

El SPP con las características descritas, compite con el Sistema Nacional de Pensiones (SNP) que es un régimen pensionario estatal que opera bajo la modalidad de reparto. Ambos regímenes son cualitativa y cuantitativamente diferentes, ya que sus contribuciones difieren (11.55\% en elcaso del SPP y $13 \%$ en el caso del SNP).

\section{Afiliación a una AFP}

Es la relación jurídica entre la Administradora de Fondo de Pensiones (AFP) y el trabajador mediante la suscripción del contrato de afiliación, con el promotor de ventas de la AFP elegida.

La afiliación del trabajador queda perfeccionada con la suscripción del contrato de afiliación, que es un contrato por adhesión, donde se establecen los deberes y derechos del afiliado y de la AFP que rigen desde el otorgamiento del CUSPP (Código Único de Identificación del Sistema Privado de Pensiones) por parte de la SBS y AFP; el formato y las cláusulas son predeterminados por la SBS y AFP.

Pueden suscribirse los trabajadores dependientes o independientes, sean peruanos o extranjeros. Los peruanos que trabajen, definitiva o temporalmente en el extranjero se afiliarán como independientes, de ser el caso.

Los regímenes previsionales en general, no es de naturaleza laboral sino de seguridad social. 
En caso que el trabajador sea dependiente, la AFP notifica a su empleador mediante un correo de notificación especial. El plazo para remitir las copias respectivas es de 4 días a partir de la generación del CUSPP.

Asimismo, dentro de los últimos 5 días de cada mes la AFP remitirá al empleador una hoja de conciliación de la totalidad de contratos enviados durante el mismo mes, con la finalidad de verificar o declarar ciertos datos, la que será devuelta por el empleador, en un plazo de 10 días de recibida.

Si por la verificación, el empleador determinase la ausencia de vínculo laboral, deberá devolver junto con la hoja de conciliación, el contrato de afiliación.

La AFP notificará al trabajador; este hecho que modifica su condición de "trabajador", de no estar conforme presentará a la AFP, las pruebas que sustenten la existencia de tal vínculo.

Si el empleador reconoce el vínculo laboral, la AFP informa este hecho al afiliado, alcanzándole en la misma oportunidad su número de CUSPP.

\section{CUSPP}

Es el Código Único de Identificación del Sistema Privado de Pensiones y es generado por la SBS y AFP.

Se asigna por única vez al trabajador cuando se incorpora al SPP y su vigencia es indefinida. La AFP procede a registrar el CUSPP en el original y copias del contrato de Afiliación.

\section{Apertura de la CiC}

Una vez que se asigna el CUISPP, la AFP deberá abrir una Cuenta Individual de Capitalización (CIC) donde se registraran los aportes al SPP.

\section{Carpeta individual del AFiLiado}

Es un expediente por cada afiliado, que contiene todos los documentos relacionados con la vida previsional del trabajador Incorporado al SPP.

\section{AFILIACIÓN CON PARTICIPACIÓN DEL EMPLEADOR}

Si un trabajador no incorporado al SPP ingresa a laborar, el empleador requerirá por escrito al tra- bajador del sistema previsional al que se encuentra incorporado, con indicación de la AFP a la que está afiliado. El trabajador que no se encuentre afiliado deberá indicar la AFP a la que desee afiliarse o su deseo de permanecer o incorporarse al SNP, lo que deberá hacerse dentro del plazo de 10 días calendarios; si lo está, deberá Indicar la AFP a la que está afiliado.

Multiafiliación Pura: Situación que se origina cuando un trabajador suscribe dos o más contratos de afiliación, recibiendo los correspondientes CUSPP.

Multiafiliación Presunta: Se origina cuando un trabajador no puede recibir el CUSPP por que ya existe un afiliado que ha registrado el mismo número de documento de identidad.

\section{Nulidad De CONTRATO}

Causales:

$$
\text { El contrato de Afiliación es declarado nulo por: }
$$

- Comprobar que la firma de trabajador es falsificada.

- En el caso de afiliación por responsabilidad del empleador, cuando no se haya cumplido con las normas que regulaban esta forma de afiliación.

- Comprobarse la inexistencia del afiliado.

- El contrato de afiliación puede ser anulado por:

- Comprobar que el afiliado venía percibiendo pensión vitalicia bajo el régimen D.L. No 19990, con anterioridad a su incorporación al SPP.

- Comprobarse que el afiliado, a la fecha de su incorporación al SPP, cumplía con los requisitos para obtener una pensión vitalicia del D. L. No 19990.

Comprobarse que el afiliado haya sido excluido de la cobertura del Sistema Nacional de Pensiones, como consecuencia de que la fecha de ocurrencia de invalidez, o sea anterior a la fecha de su incorporación al SPP, venía percibiendo pensión vitalicia bajo el régimen D.L. No 19990.

\section{Traspasos}

El afiliado puede cambiar de AFP en el momento que así lo decida. Para dicho efecto, presenta ante la 
AFP a la que desea trasladarse, la solicitud correspondiente.

\section{BONO DE RECONOCIMIENTO}

El bono de reconocimiento es un título valor garantizado por el Estado emitido por la Oficina de Normalización Previsional (ONP), el que reconoce las aportaciones que realizó el trabajador al Sistema Nacional de Pensiones (sistema administrado anteriormente por el Instituto Peruano de Seguridad Social).En la actualidad, existen tres tipos de bono de reconocimiento: el bono 1992, el bono 1996 y el bono 2001. La diferencia radica en el período de aportes al SNP que cada uno reconoce a favor del afiliado.

\section{Tipos de Prestaciones}

- Deben ser nominativos.

- Deben expresarse en moneda nacional y mantener su valor constante en función al Índice de Precios al Consumidor para Lima Metropolitana que publica el INEI o el indicador que lo sustituya, tomándose como base el índice del mes de diciembre de 1992 (para el bono de 1991), enero de 1997 (para el bono de 1996) y el Índice de enero 2002 (para el bono 2001).

\section{Documentos Sustentatorios}

- Boletas de pagos emitidos legalmente.

- Liquidación por Tiempo de Servicios.

- Póliza de seguro de vida.

- Certificado de Retenciones del Impuesto a la Renta de Quinta categoría.

- Comprobante de pago de aportaciones, para los períodos de aportación como facultativo.

- Declaración jurada del empleador.

\section{Documentos adicionales}

- Certificados de Trabajo.

- Constancia de Remuneraciones y Descuentos.

- Planilla de Remuneraciones.

- Otros similares.
- Copia simple de su DNI, carné de extranjería o partida de nacimiento legible.

\section{Ventajas Legales PARA AFILIAdos}

Hay varios hechos relevantes que se han producido en los últimos dos años y que han significado una mejora evidente para el sistema privado de pensiones entre ellos, señalamos los siguientes:

- La jubilación anticipada para trabajadores afiliados al SPP que realizan labores de alto riesgo.

- Incorporación a la Superintendencia de Banca y Seguros el control de las AFP.

- Se han promulgado nuevas normas sobre calificación y clasificación de riesgo de inversiones, para el financiamiento de concesiones, estableciéndose también los instrumentos elegibles, requerimientos y modalidades para esta inversión.

- La transferencia al exterior de fondos pertenecientes a afiliados que dejan el país en forma definitiva.

- El establecimiento de una pensión mínima para equiparar los beneficios de los sistemas privados y públicos.

- Se ha permitido la jubilación adelantada del régimen del Decreto Ley No 19990 para aquellos afiliados que optaran por afiliarse a una AFP.

- Un régimen especial de jubilación anticipada para desempleados que tengan más de 55 años de edad y 12 meses sin empleo.

- La actualización de los bonos de reconocimiento hasta el año en curso, reconociendo así los aportes efectuados por los trabajadores al Sistema Nacional de Pensiones, eliminando los requisitos de tiempo de aportación mínima para afiliarse a una AFP.

- La administración de fondos múltiples que permitan a las AFP ofrecer diferentes productos de acuerdo al perfil de inversiones que prefieran los afiliados para su ahorro previsional. La idea es combinar, en razón de la edad de los afiliados, productos de mayor riesgo pero también de mayor rendimiento cuando el afiliado es joven, para luego optar por productos de menor riesgo cuando el afiliado llega a una determinada edad. 


\section{CONCLUSIONES}

1. Cobro elevado de comisiones.

2. No hay transparencia de información.

3. La mayoría de usuarios no determina qué tipo de pensión le conviene.

4. Algunas de las AFP, no tienen la información precisa de la tasas que cobrarán, y en el tiempo es más difícil de calcular.

5. Para el cambio de una AFP a otra, hay muchos incentivos y facilidades. Sin embargo, si el afiliado no participa es cambiado automáticamente.

6. En la calculadora que proporciona la S.B.S., para que el afiliado estime lo que más le conviene, se observa que las tasas son más bajas, e históricamente, luego que es capturado el afiliado, éstas suben.

\section{RECOMENDACIONES}

1. Marcar pautas de control interno para alcanzar mejoras en las pensiones y extender la cobertura del sistema, sin descuidar la estabilidad de las finanzas públicas.

2. Los fondos públicos deben dirigirse prioritariamente a crear una pensión asistencial para los ancianos pobres, que es perfectamente financiable.

3. Que exista varios sistemas de pensiones, evitando el monopolio.

\section{REFERENCIAS BIBLIOGRÁFICAS}

1. Fred R. David.-Conceptos de administración estratégica.-Novena edición-Pearson educación Prentice Hall, México, 2008.

2. Kaplan Robert S. y Norton David P. Cuadro de Mando Integral. Ediciones Gestión 2001.

3. Los Sistemas de Pensiones en el Perú-Ministerio de Economía y Finanzas. Dirección General de Asuntos Económicos y Sociales. Pág. 4-36.

4. Página de internet de la SBS - Superintendencia de Banca y Seguro y AFP.
5. Página de internet de la SBS - Derechos del afiliado.

6. Diario Gestión.

7. Diario La República.

8. Diario El Comercio.

9. Comentarios de diversos diarios, sobre AFP'sEnero a Julio 2013.

\section{REFERENCIAS ELECTRÓNICAS}

1. Beneficios del Sistema Privado de Pensiones. Recuperado el 6 de noviembre de 2010, de: http:// www.slideshare.net/peruasociacionafp/100823aafp-beneficios-del-sistema-privado-de-pensiones.

2. Doce razones para afiliarse a una AFP. Recuperado el 6 de noviembre de 2010, de http://cies. org.pe/files/ES/bol50/Rojas.pdf.

3. El Sistema Privado de Pensiones y su papel en la economía peruana. Recuperado el 7 de noviembre de 2010, de: http://www.sbs. gob.pe/ app/ stats/EstadisticaBoletinEstadistico.asp? $\mathrm{p}=31$ \#

4. El Sistema Nacional de Pensiones. Recuperado el 8 de noviembre de 2010, de http: / / www.revistaactualidadlaboral.com/ biblioteca/legislacion/ legisactualidad/dl19990.htm.

5. Ministerio de Economía y Finanzas - Dirección General de Asuntos Económicos y Sociales (Mayo, 2004). Recuperado el 11 de noviembre de 2010, de http://www.mef.gob.pe/ESPEC/ sistemas_pensiones.pdf.

6. Paz Panizo, Jorge y Ugaz Vallenas, Rafael (Marzo, 2003).Análisis del Sistema Privado de Pensiones. Recuperado el 10 de noviembre de 2010, de http://www.consorcio.org/CIES/html/pdfs/ PB0109.pdf.

7. Quino Sandoval, Dulce. Sistema nacional de Pensiones. Recuperado el 8 de Noviembre de 2010 de: http://www.monografias.com/trabajos74/ decreto-sistema-seguridad-social-pensiones/decreto-sistema-seguridad-social-pensiones $2 . s h t m l$. 
8. Rodríguez Herrera, Adolfo y Durán Valverde, Fabio (Julio, 2000) Costos e incentivos en la organización de un Sistema de Pensiones. Recuperado el 5 de noviembre de 2010, de http:// www.eclac.org/ publicaciones/xml/7/4907/ lcl1388e.pdf.
9. Taller de Capacitación Descentralizado: Sistemas Administrativos, Controly Gestión Fiscal (Junio de 2006). Los Sistemas de Pensiones en Perú. 